\title{
Acknowledging iJOE 2017 Reviewers
}

\author{
https://doi.org/10.3991/ijoe.v14i02.8407
}

We like to extend much gratitude to the individuals who served as manuscript reviewers for the International Journal of Online Engineering (iJOE) Volume 13 (2017). Our reviewers generously volunteer their time, and although intangible benefits may be gleamed from serving in the capacity of a reviewer, reviewers are also faced with increased demands on their time to complete professional activities. We are grateful for their time, effort and dedication.

A. Y. Al-Zoubi, Princess Sumaya University for Technology Amman, Jordan Abdul K.M. Azad, Northern Illinois University, USA

Alberto Cardoso, University of Coimbra, Portugal

Alexander Kist, University of Southern Queensland, Australia

Alexander Zimin, Bauman Moscow State Technical University, Russia

Andreas Pester, Carinthia University of Applied Sciences, Austria

Antonio Batista, Instituto de Plasmas e Fusao Nuclear, Portugal

Bin Cao, Hebei University Of Science and Technology, China

Celina P Leão, University of Minho, Portugal

Chenyu Wang, University of Houston. USA

Chuang Ma, Shanghai Polytechnic University, China

Cornel Samoila, Transilvania University Brasov, Romania

Ding Yu, University of Houston, USA

Donald Reyes, San Mateo County Community College District, San Mateo, USA

Doru Ursutiu, University Transilvania of Brasov, Romania

Dr. Hao Qian, Rovi Corporation, USA

Feng Li, Henan University of Science and Technology, China

Filomena Soares, University of Minho, Portugal

Frode Eika Sandnes, Akershus University College of Applied Sciences, Norway

Gong Binlsung, National Chi Nan University, Taiwan, ROC

Guangdong Tian, Jilin University, China

Guanxin Yao, Nanchang University, China

Haibin Lv, Huanhai Marine Engineering Prospecting Institute, China

Haidi Ibrahim, Universiti Sains Malaysia

Hatem Hamad, The Islamic University of Gaza

Hélia Guerra, University of Azores, Portugal

Horácio Fernandes, Instituto Superior Técnico de Lisboa, Portugal

Huaizhong Li, Lishui University, China

Ian Grout, University of Limerick, Ireland

Isidro Calvo, University of the Basque Country, Spain

James Wolfer, Indiana University South Bend, USA

Javier Zubia, University of Deusto, Spain

Jiacun Wang, Monmouth University, USA

Jian Wang, Shanghai Polytechnic University, China

Jie Xiong, Yangtze University, China

Jinchun $\mathrm{Hu}$, Tsinghua University, China

Juarez Bento Silva, Universidade Federal de Santa Catarina, Brazil

Junxiao Zhu, University of Houston, USA

Kai Zheng, Chongqing University of Posts and Telecommunications, China 
Kalyan Ram Bhimavaram, Electrono Solutions Pvt Ltd, India Karsten Henke, Ilmenau University of Technology, Germany Katarina Zakova, Slovenská technická univerzita v Bratislave, Slovakia Lane Thames, Georgia Institute of Technology, USA

Liang Peng, Utah State University, USA

Lusi de La Torre, UNED, Spain

Mansharipova Arkel, SIEMENS Co. Ltd., Shanghai, China

Michael E. Auer, CTI Frankfurt/Main, Germany

Mikulas Huba, Slovak University of Technology, Slovakia

Na Wang, Shanghai Polytechnic University, China

Nei Bo, Longqiao College, Lanzhou, China

Ning Liu, Illinois Institute of Technology, USA

Ning Wang, Texas Southern University, USA

Ning Wang, University of Houston, USA

Niu Tie, Inner Mongolia University, China

Olawale B. Akinwale, Obafemi Awolowo University, Nigeria

Pablo Orduña, LabsLand, Spain

Paulo Menezes, University of Coimbra, Portugal

Petru Adrian Cotfas, Transilvania University Brasov, Romania

Radojka Krneta, University of Kragujevac, Serbia

Raymundo Buenrostro, University of Colima, Mexico

Reinhard Langmann, Hochschule Düsseldorf, Germany

Ricardo Costa, ISEP/LABORIS, Portugal

Riko Safaric, University of Maribor, Slovenia

Roderval Marcelino, Universidade Federal de Santa Catarina, Brazil

Ruben Heradio, UNED, Spain

Ruihang Huang, South China Normal University, Guangzhou, China

Sami Habib, Kuwait University

Sebastian Dormido, Universidad Nacional de Educación a Distancia, Spain

Tareq Alhmiedat,Zarqa University, Jordan

Thomas L Lago, QirraSound, Sweden

Thrasyvoulos Tsiatsos, Aristotele University of Thessalonik, Greece

Tingwen Huang, Texas A\&M University at Qatar, Qatar

Tonglin Li, Illinois Institute of Technology, USA

Wang Ning, Texas Southern University, USA

Wen Yu, National Polytechnic Institute, Mexico

Xiaoguang Yue, Wuhan University of Technology, China

Xiaojian Zhu, Southeast University, China

Xiaoming Hu, Shanghai Polytechnic University, China

Xuemin Chen, Texas Southern University, USA

Yu Ding, University of Houston, USA

Yu Zhang, East China University of Science and Technology, China

Zhihan Lv, Chinese Academy of Science, China

Zhou Zhou, Salesforce, USA

Michael E. Auer and Abul K. M. Azad

Editors-in-Chief

International Journal of Online Engineering 